\title{
USING OF THE SPSA METHOD TO IMPROVE THE ACCURACY OF UAV FOLLOWING A GIVEN ROUTE UNDER THE ACTION OF WIND LOADS
}

\author{
Konstantin Amelin \\ Laboratory "Control of Complex Systems" \\ IPME RAS \\ SEC "Mathematical robotics and artificial intelligent" \\ Saint Petersburs State University \\ Russia \\ k.amelin@spbu.ru
}

\author{
Vladimir Maltsev \\ The Faculty of Mathematics and Mechanics \\ Saint Petersburs State University \\ Russia \\ st054439@ student.spbu.ru
}

Article history:

Received 08.11.2021, Accepted 01.12.2021

\begin{abstract}
In the modern world, UAVs (unmanned aerial vehicles) are increasingly used in everyday life in solving civilian tasks. One of the main applications of UAVs is data collection with their reference to a given coordinate system. For example, for the task of aerial photography, it is necessary to accurately link each image to the global coordinate system. In addition to the exact location of coordinates, it is worth the exact movement of a given route, to collect data of exactly those places that are needed. Thus, it is very important to ensure the minimum deviation of the UAV from the given route under the conditions of external disturbances (wind disturbances) acting on it. The article describes a procedure for assessing wind speed and direction for a UAV control system using the SPSA method. The simulation results of the algorithm operation, confirmed during flight tests on an ultralight UAV with an ardupilot autopilot, are presented.
\end{abstract}

\section{Key words}

UAV control system, randomized algorithms, Kalman filter, GNSS, random process prediction methods.

\section{Introduction}

Currently, unmanned aerial vehicles (UAVs) are increasingly used for various tasks. To perform an autonomous flight, the UAV's onboard autopilot memory records the flight route in the form of a set of points in space, which is used for navigation. Successful performance of applied tasks, such as conducting aerial photography, depends on the accuracy of positioning and compliance with the specified trajectories of movement along the route. UAV uses data of one or several global navigation satellite systems (GNSS) GPS (USA),
GLONASS (Russia), BeiDou (China), Galileo (European Union) in combination with data of inertial navigation system (INS) to determine position in space.

The INS includes a set of sensors that use the inertial properties of the vehicle (linear acceleration, angular velocity, and magnetic azimuth) to track and determine its position in space. Due to the limitations on power consumption, size and weight imposed on the onboard UAV equipment, onboard INS have relatively low accuracy and accumulate significant error over time, so their application is limited to maintaining the current equilibrium. For positioning, only the data from the global navigation system sensor is used, which comes at discrete points in time and often contains errors. The dynamic characteristics of UAV and quality of operation of sensors installed on it also have a significant influence on the flight. At the same time, there are external random influences on the UAV in the form of wind loads, so the direction of its movement and speed of the vehicle can fluctuate. To smooth the resulting errors, various linear filters are used, among which the most popular is the Kalman filter and its variations [Ribeiro and Ribeiro, 2004]. However, such filters are not good enough to cope with errors whose mathematical expectation is not equal to zero [Palanisamy et al., 2015]. The wind effects also lead to the appearance of biased error.

One way to improve the performance of the Kalman filter in such a situation is to use randomized methods [Duník et al., 2011]. In particular, the Simultaneous Perturbation Stochastic Approximation (SPSA) method [Granichin, 1992; Spall, 1992; Spall, 2003] is widely used. It is supposed to deliberately remove the system from the balance state in order to counteract random influences. This method has a mathematical structure that allows to estimate its stochastic properties also for optimization problems subject to noise and uncertainties. 
Due to these properties, SPSA is used to optimize many adaptive control problems, such as UAV group control [Antal et al., 2010] and the tuning of complex, largescale models [Alessandri and Parisini, 1997].

The paper proposes to extend the results obtained in [Amelin and Granichin, 2011; Amelin, 2012; Amelin and Granichin, 2012; Amelin and Granichin, 2016] for the application of the SPSA method to counteract random changes in wind strength and direction, and to develop a wind estimation module based on this method for testing on a real UAV.

\section{Methods of Following the UAV Trajectory}

\subsection{Algorithms for Maintaining a Trajectory}

Accurate trajectory tracking is one of the main requirements for UAVs [?].

Strategies for solving the problem of following a given path can be categorized into two groups: geometric and theoretical.

Geometric methods represent target point pursuit [Conte et al., 2004], Line-Of-Sight (LOS) guidance laws [Rysdyk, 2006], and their combinations [Kothari et al., 2010]. Such algorithms use Virtual Target Point (VTP). The guidance laws instruct the UAV to pursue the VTP, which eventually leads it to a given trajectory. The distance between the VTP and the UAV position projected on the trajectory is called the virtual distance. The stability of the LOS guidance laws depends significantly on the choice of the virtual distance parameter. The pursuit and line-of-sight guidance laws can be combined to create a new guidance law for path following [Kothari et al., 2010]. Instead of using line-of-sight pursuit or guidance, the Non-Linear Guidance Law (NLGL) was developed in [Park et al., 2007] using VTP. Usually, path following laws are derived from Lyapunov stability analysis, which guarantees globally stable convergence to the desired path. Another variant of vector field is proposed in [Chen et al., 2013], which is called tangent vector field induction. A development of this method capable of handling slow wind changes is given in [Bingyu Zhou et al., 2017].

Theoretical control methods, in particular nonlinear control methods, are popular for path tracking applications. They provide some robustness to wind disturbances. A common approach used in path tracking based on Proportional-Integral-Differential (PID) control [Sun et al., 2008], but does not work as well as NLGL [Park et al., 2007].

Several other types of theoretical control methods are known, which have been developed to solve the problems of UAV following a given route. Some of them are well known methods are linear-quadratic control (LQR) [Lee et al., 2010], sliding mode control [Healey and Lienard, 1993], control with model prediction [Jackson et al., 2008], step-back control [Ahmed and Subbarao, 2010], gain scheduling theories [Cunha et al., 2003] and dynamic programming [da Silva and de Sousa, 2010], and control based on piecewise affine functions [Shehab and Rodrigues, 2005].

\subsubsection{Error Filtering for Offset Measurement}

Errors or uncertainties in the control signal usually represent a random process. The problem of prediction of such a random process is most typical for Kalman filtering, which is based on the works of R.E. Kalman and R.S. Busey [Kim and Bang, 2018]. In addition to statistical ones, there are minimax problem formulations. They assume that uncertainties are only bounded in some sense, otherwise they can be arbitrary. In such formulations, with a predetermined level of perturbations, one obtains predictions in the form of sets whose sizes stabilize with time [Garulli et al., 2003]. In this case, there is no way to obtain reasonable exact estimates. Further practical use of set estimators leads to complex robust stability problems [Kuntsevich, 2007].

The error detection problem is complicated by the low diversity of input data. The possibility of adding a particular signal in the control system can alleviate the problem of reconstructing unknown parameters. For example, in [Aleksandrov and Orlov, 2009], a series of single pulses in the input channel allowed the reconstruction of the impulse function of the object. In addition, special randomized signals in the input channel make it possible to determine the parameters of the control object, when considering an object model with almost arbitrary additive disturbances. The procedure proposed in [Granichin and Polyak, 2003] works for any interference and does not require a priori knowledge of its characteristics. The recovery of unknown parameter values is provided by the properties of randomized test signals, which are added in the control loop to the adaptive control own signals coming from the feedback.

\subsection{Methods of Measuring Wind Parameters Dur- ing the UAV's Flight}

Several methods are used to estimate wind parameters during UAV flight, which can be divided into two groups: with the use of special sensors and without them.

One method of measuring three-dimensional wind is the multi-hole-probe algorithm (MHPA) [Van et al., 2008], combined with data on the orientation and position of the UAV in space, and its speed. The achievable high resolution and accuracy of this method requires accurate and fast INS, as well as pressure measurements using multi-hole probes. The Pitot Tube Algorithm, (PTA) is described in [Dobrowolski et al., 2005], which does not require a multi-hole probe, but only a static Pitot tube to measure dynamic pressure, making it less complex and expensive. Wind speed can be estimated without on-board airflow sensors, for example using the NFSA (no-flow-sensor algorithm) published in [Mayer et al., 2012]. The NFSA uses only the information about the track speed and azimuth of the flight path obtained from the GNSS. Methods without special sensors are 
more complex, but cost-effective compared to the previous ones. The fixed wing UAV considered in this paper uses the Pitot tube algorithm as a compromise between cost, complexity and accuracy of the data obtained.

\section{Adaptive Randomized Algorithm}

This section presents a brief overview of the basic concepts associated with the SPSA algorithm; more technical details are described in the relevant literature [Spall, 2003].

Let us consider the problem of finding the minimum of a differentiable loss function:

$$
L_{n}(\theta): R^{p} \rightarrow R
$$

(the lower index $n$ is used to show that the loss function is affected by noise, whose distribution must satisfy several important conditions [Spall, 2003]).

The task is to find an approximation $\theta$ at which $L_{n}(\theta)$ is minimal. The SPSA method computes the approximation $\theta$ at iteration $k+1$ as follows:

$$
\theta_{k+1}=\hat{\theta}_{k}-a_{k} \hat{g}_{k}\left(\hat{\theta}_{k}\right),
$$

where $\hat{g_{k}}$ is the estimated gradient at the $k$-th iteration and $a_{k}$ is the coefficient that is planned to decrease with each iteration:

$$
a_{k}=\frac{a}{(k+A)^{\alpha}},
$$

where $a, A$ and $\alpha$ are preselected configuration coefficients. To estimate $\hat{g}_{k}(\cdot)$ the following method of "simultaneous perturbations" is used. Let $\Delta_{k}=$ $\left[\Delta_{k 1} \Delta_{k 2} \ldots \Delta_{k p}\right]^{T} \in R^{p}$ be a vector of independent random variables with zero mean (satisfying the conditions described in [Spall, 2003]), and let the sequence of vectors $\Delta_{k}$ be an independent sequence with $\Delta_{k}$ independent of $\hat{\theta}_{j}, j=0,1, \ldots, k$. The basic SPSA method (bSPSA) computes two new points in the decision space and estimates the corresponding losses as follows:

$$
\begin{gathered}
y_{k}^{+}=\hat{L}_{n}\left(\theta_{k}+c_{k} \Delta_{k}\right) \\
y_{k}^{-}=\hat{L}_{n}\left(\theta_{k}-c_{k} \Delta_{k}\right),
\end{gathered}
$$

where $c_{k}$ is a sequence of coefficients:

$$
c_{k}=\frac{c}{(k+1)^{\gamma}},
$$

where $c$ and $\gamma$ are positive configuration coefficients. Then the estimate of the gradient at the $k-t h$ iteration is calculated by the following formula:

$$
\hat{g}_{k}\left(\hat{\theta}_{k}\right)=\frac{y_{k}^{+}-y_{k}^{-}}{2 c_{k}}\left[\Delta_{k 1}^{-1} \Delta_{k 2}^{-1} \ldots \Delta_{k p}^{-1}\right]^{T} .
$$

Note that all elements of the vector $\theta$ are perturbed simultaneously, and that only two loss measures are needed to estimate the gradient independently of the size $\hat{\theta}$. In practice, the sequence $\Delta_{k}$ is independently generated by a Bernoulli distribution yielding \pm 1 with probability $\frac{1}{2}$ for each value. In this case the perturbations have the same amplitude for all components of $\theta$.

\section{Estimation of Wind Parameters Using SPSA}

\subsection{The Coordinate System}

Three Cartesian coordinate systems can be used for wind estimation according to Boyffer [Boiffier, 1999] (see Fig.1). The first is the Earth coordinate system or global coordinate system with the index $e$. It has the $i_{e}^{1}$ axis pointing north, the $i_{e}^{2}$-axis pointing east, and the $i_{e}^{3}$ - axis pointing down. The second is a connected or local coordinate system with the index $l$. When it is used, the origin is in the center of gravity of the aircraft; the $i_{l}^{1}$ axis points forward, the $i_{l}^{2}$-axis points toward starboard and the $i_{l}^{3}$-axis points downward. And the third is the velocity coordinate system with the index $s$, which is referenced to the airspeed of the UAV, that is, its speed relative to the air. It has the same origin as the local coordinate system, the $i_{s}^{1}$-axis is located along the air flow, the $i_{s}^{2}$-axis is in the UAV symmetry plane and is perpendicular to the flow and the $i_{s}^{3}$-axis is directed down relative to the UAV.

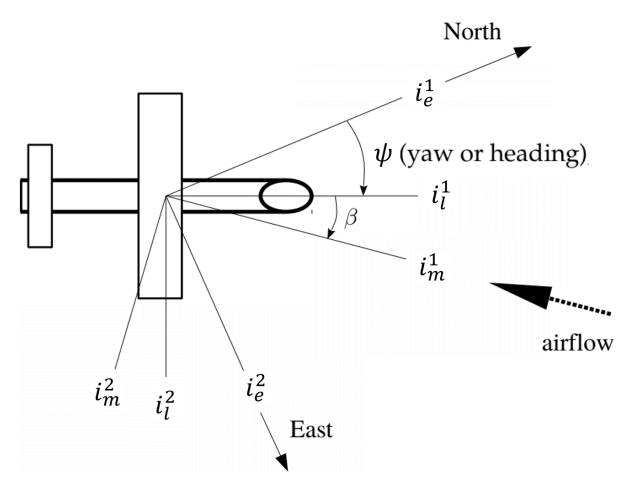

Figure 1. Top view of the wind measurement with indices $s, l$ and $e$ representing the velocity, local and global coordinate systems, respectively. $\psi$ is the yaw angle or true course of the $\mathrm{UAV}$ and $\beta$ is the side slip angle between the velocity coordinate system and the local coordinate system. 


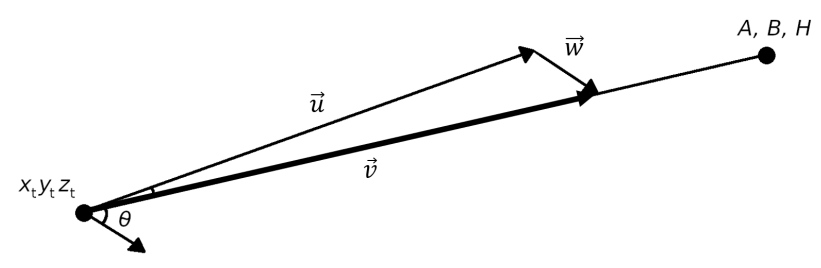

Figure 2. Target heading, course, wind drift.

\subsection{Wind Load Vector Calculation}

The vector $\vec{w}$ represents the direction and strength of the airflow. A non-stationary observer, for example, a flying UAV, sees only the relative speed $\vec{u}$, but from a fixed observation point, for example, relative to the global coordinate system. The UAV moves at a velocity of $\vec{v}$, which is the sum of $\vec{u}$ and $\vec{w}$. This ratio is the basis of all UAV wind measurement methods. The wind vector in the global coordinate system is the difference between the vectors $\vec{w}_{e}$ and $\vec{u}_{e}$. The UAV velocity vector $\vec{w}_{e}$ is usually measured using GNSS data and can be measured with fairly good accuracy, whereas the true airspeed vector $\vec{u}_{e}$ relative to the UAV is a more difficult parameter to estimate, as is the airborne position, such as in Euler angles. According to Bange [Bange, 2009], the wind vector can be calculated using the following formula:

$$
\vec{w}_{e}=\vec{v}_{e}+\mathbf{T}_{e l}\left(\vec{u}_{l}+\vec{\Omega}_{l} \times \vec{O}\right)
$$

where $\vec{u}_{l}$ represents true airspeed vector in the local coordinate system of the UAV, $\mathbf{T}_{e l}$ is a matrix of transformation from global to local coordinate system, $\vec{\Omega}_{l}$ is a vector of local angular velocities of the UAV, whilst $\vec{O}$ is a lever of the vector arm $\vec{\Omega}_{l}$, representing the distance between the INS and the airspeed sensor.

\subsection{Randomization for Estimating Unknown Wind Parameters in UAV Control}

4.3.1 Problem Statement An algorithm for calculating corrections to the course of motion using the SPSA method is proposed in [Amelin, 2012] when the wind acts with constant speed but variable direction in the horizontal plane. In this paper, to calculate the corrections to the control, we consider the impact of wind loads in the horizontal plane, where it is expressed in the deviation of the UAV from a given trajectory, and in the vertical, where due to its impact there is a deviation from the held altitude. To be able to separate the known quantities from the unknown, let us represent as components $\vec{v}_{e}=\left(v_{i_{e}^{1}}, v_{i_{e}^{2}}, v_{i_{e}^{3}}\right)^{T}$ and $\vec{w}_{e}=\left(w_{i_{e}^{1}}, w_{i_{e}^{2}}, w_{i_{e}^{3}}\right)^{T}$. For convenience, we write the horizontal wind components $w_{i_{e}^{1}}$ and $w_{i_{e}^{2}}$ in polar coordinates $\theta, b$, where $\theta$ is the wind direction and $\mathrm{b}$ is its strength. We denote the vertical wind component $w_{i_{e}^{3}}$ by $h$. The location data are computed at time intervals $\Delta$, i.e., at time $T_{k}=T_{0}+\Delta_{t}$ the triples of numbers $\left(x_{t}, y_{t}, z_{t}\right)$ arrive. To control the
$\mathrm{UAV}$, creating a sequence of control signals fed to the actuators, it is necessary to estimate the unknown parameters $\theta, b$ and $h$ from the observations $\left(\hat{x}_{t}, \hat{y}_{t}, \hat{z}_{t}\right)$.

Let a point of direction to the $\operatorname{target}(A, B, H)$ be given. At each time moment $t$, the UAV is at the point $\left(x_{t}, y_{t}, z_{t}\right)$ (see Fig.2). At each beat of time from $\mathrm{t}$ to $\mathrm{t}+1$ the course to be followed by the UAV is calculated. At this time interval the UAV movement in the direction of course $u$ is interfered by the wind.

Changes in wind direction angle and wind speed can be estimated by the following formulas:

$$
\begin{gathered}
\theta_{t+1}=\theta_{t}+\varepsilon_{t+1} \\
b_{t+1}=b_{t}+\xi_{t+1} \\
h_{t+1}=h_{t}+\mu_{t+1},
\end{gathered}
$$

where $\varepsilon, \xi$ and $\mu$ are independent, centered and equally distributed random variable quantities. The optimization of motion to the endpoint requires at time $t$ by the sequence of observations $\left(\hat{x}_{i}, \hat{y}_{i}, \hat{z}_{i}\right)_{i=0}^{t}$, to propose a model to estimate $\theta_{t+1}, b_{t+1}, h_{t+1}$, minimizing the standard deviations:

$$
\begin{aligned}
& E\left\{\left(\theta_{t+1}-\hat{\theta}_{t+1}\right)^{2}\right\} \rightarrow \min \\
& E\left\{\left(b_{t+1}-\hat{b}_{t+1}\right)^{2}\right\} \rightarrow \min \\
& E\left\{\left(h_{t+1}-\hat{h}_{t+1}\right)^{2}\right\} \rightarrow \min .
\end{aligned}
$$

\subsubsection{Algorithm for Estimating Wind Parameters} Based on the SPSA algorithm and the wind direction estimaton algorithm described in [Amelin et al., 2013], we construct estimates for the three wind parameters.

At each iteration of the autopilot operation, we calculate the heading $\psi_{t}$, which must be held at the next time beat to reach the given trajectory. Also, at each iteration, the current direction of the UAV $\phi_{t}$ is known, and consequently the error in the course $\varepsilon_{t}^{\theta}$. The distance to a given trajectory $\varepsilon_{t}^{b}$ and the distance to a given altitude $\varepsilon_{t}^{h}$ :

$$
\begin{gathered}
\varepsilon_{t}^{\theta}=\psi_{t}-\phi_{t} \\
\varepsilon_{t}^{b}=\operatorname{distance}\left(\left(x_{t}, y_{t}\right), \operatorname{line}\left(A_{n}, B_{n}, A_{n+1}, B_{n+1}\right)\right. \\
\varepsilon_{t}^{h}=a b s\left(z_{t}-H\right) .
\end{gathered}
$$

The following randomized algorithm is constructed to estimate the wind parameters: 
1. $\hat{\theta}_{0}=\hat{b}_{0}=\hat{h}_{0}=0, \alpha_{1}, \alpha_{2}, \alpha_{3}, \beta>0$.

2. Reading UAV flight variables values.

3. Generation of a sequence of $\Delta_{n}$ independent, equally distributed random variables equal to $\pm \beta$ with equal probability $\frac{1}{2}$, called trial randomized perturbations.

4. Forming the control:

$$
u_{t}=\hat{u}_{t-1}+\Delta_{t}
$$

5. Forming the estimation of changes in wind parameters:

$$
\begin{gathered}
\hat{\theta}_{t+1}=\hat{\theta}_{t}-\alpha_{1} \Delta_{t 1} \varepsilon_{t}^{\theta} \\
\hat{b}_{t+1}=\hat{b}_{t}-\alpha_{2} \Delta_{t 2} \varepsilon_{t}^{b} \\
\hat{h}_{t+1}=\hat{h}_{t}-\alpha_{3} \Delta_{t 3} \varepsilon_{t}^{h} .
\end{gathered}
$$

6. Publication of the received wind forecast for transmission to the position estimation module. Get the control $\hat{u}_{t}$.

7. Going to step 2.

\section{Approbation on an Airplane-type UAV}

\subsection{UAV Control System}

The hardware of the control system is based on a threelevel architecture [Amelin and Kaliteevskiy, 2017], which is based on the principle of dividing control algorithms into a group of onboard microcomputers. This division is based on real-time distribution of controls into required and not required work. In the proposed system, control is carried out by a group of three microcomputers: the Px4 autopilot, which operates in real time and is responsible for stabilizing and keeping the UAV in the air, as well as performing the movement mission; the Raspberry pi 4 microcomputer, which is responsible for collecting and integrating data with various sensors, as well as for generating and sending new autopilot missions; Intel graphics processor, which is responsible for processing data from stereo cameras. Microcomputers are connected by means of Serial Port.

\subsection{Autopilot Architecture}

The PX4 autopilot architecture consists of two layers: an intermediate layer and a high-level layer. The middle layer provides support for autonomous robotic systems, internal and external communications, and system integration. The high-level layer is the flight stack, which is responsible for flight control. The drone control algorithms that PX4-Autopilot supports are in the same codebase. The system has a reactive design. This means that all functionality is divided into components that can be replaced or reused, communication between components occurs through asynchronous messages, and the system is able to cope with different workloads.

In this work, the wind estimation module for the PX4Autopilot was implemented. It starts in a common work queue with a frequency of $10 \mathrm{~Hz}$. At each iteration, the following messages are read:

1. vehicle attitude - data on the orientation of the UAV in space;

2. vehicle local position - UAV local position;

3. vehicle gps position - data from the navigation system;

4. vehicle global position - UAV position in global coordinates;

5. airspeed validated - airspeed data;

6. position setpoint triplet - previous, current and next navigation points.

To publish the wind estimate obtained during the work of this module, the wind prediction message was created and processed in the position estimation module.

\subsection{Testing on Real UAV}

The randomized algorithm was tested on an UAV planner type with PX4 autopilot. The algorithms was tested in case the fain change of wind. Two flights were performed: using the randomized wind estimation module and the original module. The UAV using the wind estimation module is not inferior to control without it, and sometimes allows to follow the trajectory more accurately. The average deviation from the trajectory when flying without the wind estimation module was 1.2 meters, and with the wind estimation module it was less 0.8 meters.

\section{Conclusion}

The applicability of the randomized algorithm in the UAV trajectory deviation estimation module was investigated. A method for accounting for wind parameters using the SPSA method was proposed. A software module for estimation-based corrections for the PX4 autopilot was developed. The algorithm was tested on an UAV. The flight results showed that the application of the new estimation method is not worste than PX4 algoriphm. In future we plan to test the UAV with strong changes of wind.

\section{Acknowledgements}

Sections 1,4-5 were supported by the IPME RAS by Russian Science Foundation (project no. 21-19-00516). Section 2-3 was supported by the St. Petersburg State University (project No. 73555239). 


\section{References}

Ahmed, M. and Subbarao, K. (2010). Nonlinear 3-d trajectory guidance for unmanned aerial vehicles. In 2010 11th International Conference on Control Automation Robotics Vision, pp. 1923-1927.

Aleksandrov, A. and Orlov, Y. (2009). Finite-frequency identification: dynamic algorithm. Control problems, pp. 2-8.

Alessandri, A. and Parisini, T. (1997). Nonlinear modeling of complex large-scale plants using neural networks and stochastic approximation. IEEE Transactions on Systems, Man, and Cybernetics - Part A: Systems and Humans, 27 (6), pp. 750-757.

Amelin, K. (2012). Randomization in control for a small uav fly optimization under unknown arbitrary wind disturbances. Cybernetics and Physics, 1(2), pp. 7988.

Amelin, K., Amelina, N., Granichin, O., Granichina, O., and Andrievsky, B. (2013). Randomized algorithm for uavs group flight optimization. IFAC Proceedings Volumes, $\mathbf{4 6}$ (11), pp. 205-208.

Amelin, K. and Granichin, O. (2011). Potential of randomization in a kalman-type prediction algorithm at arbitrary external noise in observations. Gyroscopy and Navigation, 2 (4), pp. 277-284.

Amelin, K. and Granichin, O. (2012). Randomized controls for linear plants and confidence regions for parameters under external arbitrary noise. In 2012 American Control Conference (ACC), pp. 851-856.

Amelin, K. and Granichin, O. (2016). Randomized control strategies under arbitrary external noise. IEEE Transactions on Automatic Control, 61 (5), pp. 13281333.

Amelin, K. and Kaliteevskiy, V. (2017). Inverse kinematics in ultralight uav control problem with additional on-board microcomputer. Cybernetics and Physics, 6, pp. 155-159.

Antal, C., Granichin, O., and Levi, S. (2010). Adaptive autonomous soaring of multiple uavs using simultaneous perturbation stochastic approximation. In 49th IEEE Conference on Decision and Control (CDC), pp. 3656-3661.

Bange, J. (2009). Coordinated Path Following for TimeCritical Missions of Multiple UAVs via L1 Adaptive Output Feedback Controllers. Sierke Verlag.

Bingyu Zhou, Satyavada, H., and Baldi, S. (2017). Adaptive path following for unmanned aerial vehicles in time-varying unknown wind environments. In 2017 American Control Conference (ACC), pp. 1127-1132. Boiffier, J.-L. (1999). Dynamics of flight: the equations. The Aeronautical Journal (1968), 103 (1025), pp. 358-358.

Chen, H., Chang, K., and Agate, C. S. (2013). Uav path planning with tangent-plus-lyapunov vector field guidance and obstacle avoidance. IEEE Transactions on Aerospace and Electronic Systems, 49 (2), pp. 840 856.
Conte, G., Duranti, S., and Merz, T. (2004). Dynamic $3 \mathrm{~d}$ path following for an autonomous helicopter. IFAC Proceedings Volumes, 37 (8), pp. 472477. IFAC/EURON Symposium on Intelligent $\mathrm{Au}-$ tonomous Vehicles, Lisbon, Portugal, 5-7 July 2004.

Cunha, R., Silvestre, C., and Pascoal, A. (2003). A path following controller for model-scale helicopters. In 2003 European Control Conference (ECC), pp. 22482253.

da Silva, J. E. and de Sousa, J. B. (2010). A dynamic programming approach for the motion control of autonomous vehicles. In 49th IEEE Conference on Decision and Control (CDC), pp. 6660-6665.

Dobrowolski, B., Kabaciński, M., and Pospolita, J. (2005). A mathematical model of the self-averaging pitot tube. Flow Measurement and Instrumentation, 16, pp. 251-265.

Duník, J., Straka, O., and Šimandl, M. (2011). The development of a randomised unscented kalman filter. IFAC Proceedings Volumes, 44 (1), pp. 8-13. 18th IFAC World Congress.

Garulli, A., Giarre, L., and Zappa, G. (2003). Identification of approximated hammerstein models in a worstcase setting. Automatic Control, IEEE Transactions on, 47, pp. 2046- 2050.

Granichin, O. (1992). Procedure of stochastic approximation with disturbances at the input. Automation and Remote Control, 53 (2), pp. 232-237.

Granichin, O. and Polyak, B. T. (2003). Randomized Algorithms of an Estimation and Optimization Under Almost Arbitrary Noises, vol. 293. Nauka.

Healey, A. J. and Lienard, D. (1993). Multivariable sliding mode control for autonomous diving and steering of unmanned underwater vehicles. IEEE Journal of Oceanic Engineering, 18(3), pp. 327-339.

Jackson, S., Tisdale, J., Kamgarpour, M., Basso, B., and Hedrick, J. K. (2008). Tracking controllers for small uavs with wind disturbances: Theory and flight results. In 2008 47th IEEE Conference on Decision and Control, pp. 564-569.

Kim, Y. and Bang, H. (2018). Introduction to Kalman Filter and Its Applications. Kim, Youngjoo and Bang, Hyochoong.

Kothari, M., Postlethwaite, I., and Gu, D.-W. (2010). A suboptimal path planning algorithm using rapidlyexploring random trees. International Journal of Aerospace Innovations, 2, pp. 93-104.

Kuntsevich, V. (2007). Robust stability and synthesis of discrete control systems for nonlinear objects. Problemy Upravleniya I Informatiki (Avtomatika), 39, pp. 5-22.

Lee, S., Cho, a., and Kee, C. (2010). Integrated waypoint path generation and following of an unmanned aerial vehicle. Aircraft Engineering and Aerospace Technology, 82, pp. 296-304.

Mayer, S., Hattenberger, G., Brisset, P., Jonassen, M., and Reuder, J. (2012). A 'no-flow-sensor' wind estimation algorithm for unmanned aerial systems. Inter- 
national Journal of Micro Air Vehicles, 4, pp. 15-30.

Palanisamy, R. p., Cho, S., Kim, H., and Sim, S.H. (2015). Experimental validation of kalman filterbased strain estimation in structures subjected to nonzero mean input. Smart Structures and Systems, 15, pp. 489-503.

Park, S., Deyst, J., and How, J. P. (2007). Performance and lyapunov stability of a nonlinear path following guidance method. Journal of Guidance, Control, and Dynamics, 30 (6), pp. 1718-1728.

Ribeiro, M. and Ribeiro, I. (2004). Kalman and Extended Kalman Filters: Concept, Derivation and Properties. jhg.

Rysdyk, R. (2006). Unmanned aerial vehicle path following for target observation in wind. Journal of Guidance, Control, and Dynamics, 29 (5), pp. 1092-1100.

Samson, C. (1993). Time-varying feedback stabilization of car-like wheeled mobile robots. Int. J. Rob. Res., 12 (1), pp. 55-64.
Shehab, S. and Rodrigues, L. (2005). Preliminary results on uav path following using piecewise-affine control. In Proceedings of 2005 IEEE Conference on Control Applications, 2005. CCA 2005., pp. 358-363.

Spall, J. (1992). Multivariate stochastic approximation using a simultaneous perturbation gradient approximation. IEEE Trans. Automat. Control, 37 (3), pp. 332341.

Spall, J. C. (2003). Simultaneous Perturbation Stochastic Approximation. J. C. Spall.

Sun, M., Zhu, R., and Yang, X. (2008). Uav path generation, path following and gimbal control. In 2008 IEEE International Conference on Networking, Sensing and Control, pp. 870-873.

Van, A., Kroonenberg, D., Martin, T., Buschmann, M., Bange, J., and Vörsmann, P. (2008). Measuring the wind vector using the autonomous mini aerial vehicle m2av. Journal of Atmospheric and Oceanic Technology - J ATMOS OCEAN TECHNOL, 25. 